\title{
Length Variation Effect of the Impulse Response Model of a Secondary Path in Embedded Control
}

\author{
Young-Sup Lee, Yunseon Choi, and Jeakwan Kim \\ Department of Embedded Systems Engineering, Incheon National University, 119 Academy-ro, Yeonsu-gu, \\ Incheon 406-772, Republic of Korea \\ Correspondence should be addressed to Young-Sup Lee; ysl@inu.ac.kr
}

Received 25 December 2015; Accepted 25 February 2016

Academic Editor: Marco Anisetti

Copyright (C) 2016 Young-Sup Lee et al. This is an open access article distributed under the Creative Commons Attribution License, which permits unrestricted use, distribution, and reproduction in any medium, provided the original work is properly cited.

This study presents theoretical and experimental investigation on the length variation effect of the impulse response function (IRF) for the secondary path model in active noise control using an embedded control board. A narrowband sweep noise was the disturbance for control in a duct with the length of $1800 \mathrm{~mm}$. The IRF model incorporated into an adaptive feedforward filtered-x LMS (FxLMS) algorithm was then analyzed in the variation of its length in terms of the mean square error, computation complexity, stability requirement, and attenuation performance before and after control. The FxLMS algorithm with various IRF lengths was implemented in a dSPACE DS1104 embedded control board for the real-time control. Finally the most reasonable IRF length, considering the computation complexity and performance, can be determined through the systematic investigation. The results in this study can be used for practical active noise control systems.

\section{Introduction}

Active noise cancellation (ANC) is a technique based on adaptive feedforward control mostly to superpose an artificial sound to an unwanted disturbance noise [1-3]. The artificial sound, which is generated through the secondary loudspeaker, has to be of the same amplitude but the exact antiphase to the primary noise for best performance. An ANC system requires a good model for the compensation of the secondary path (or the plant) when the filtered-x least mean square (FxLMS) algorithm is used. It is known that the accuracy of the secondary path model has a decisive influence along with the convergence coefficient on the performance, stability, and convergence speed of the ANC system $[1,4]$. The impulse response function (IRF) of the secondary path of the FxLMS approach is usually implemented as an FIR (finite impulse response) filter in a real-time embedded control board because the FIR filter is stable and easy to implement. An IIR (infinite impulse response) filter can be used to replace a long FIR filter in order to minimize the computation requirement, although the IIR filter can become rather easily unstable than an FIR one.
An optimum length of the IRF is critically important in terms of the computation complexity, performance, and stability of the control. The optimum length of the IRF must contain the main acoustic modes to be controlled or suppressed in the secondary path. The difference between the designed IRF and the actual secondary path causes the existence of the uncertainty of the plant $[1,5]$. This difference is increased when the plant is perturbed. Thus the length of the IRF of the secondary path is critically important in terms of stability and performance in the FxLMS algorithm.

For robust stability of the FxLMS algorithm, Ren and Kumar suggested an SPR (strictly positive real) condition by combining the secondary path and its model [6]. Fraanje et al. investigated minimum norm regularization filters which can stabilize the update algorithm to prevent instability as the performance of the algorithm can be degraded not only by the plant model uncertainty but also by an unstable update method [7]. Berkhoff examined an approach for improved stability of adaptive feedforward controllers without measuring the plant uncertainty [8].

In this study, the length variation of the IRF of the secondary path is considered to analyze the control performance, 


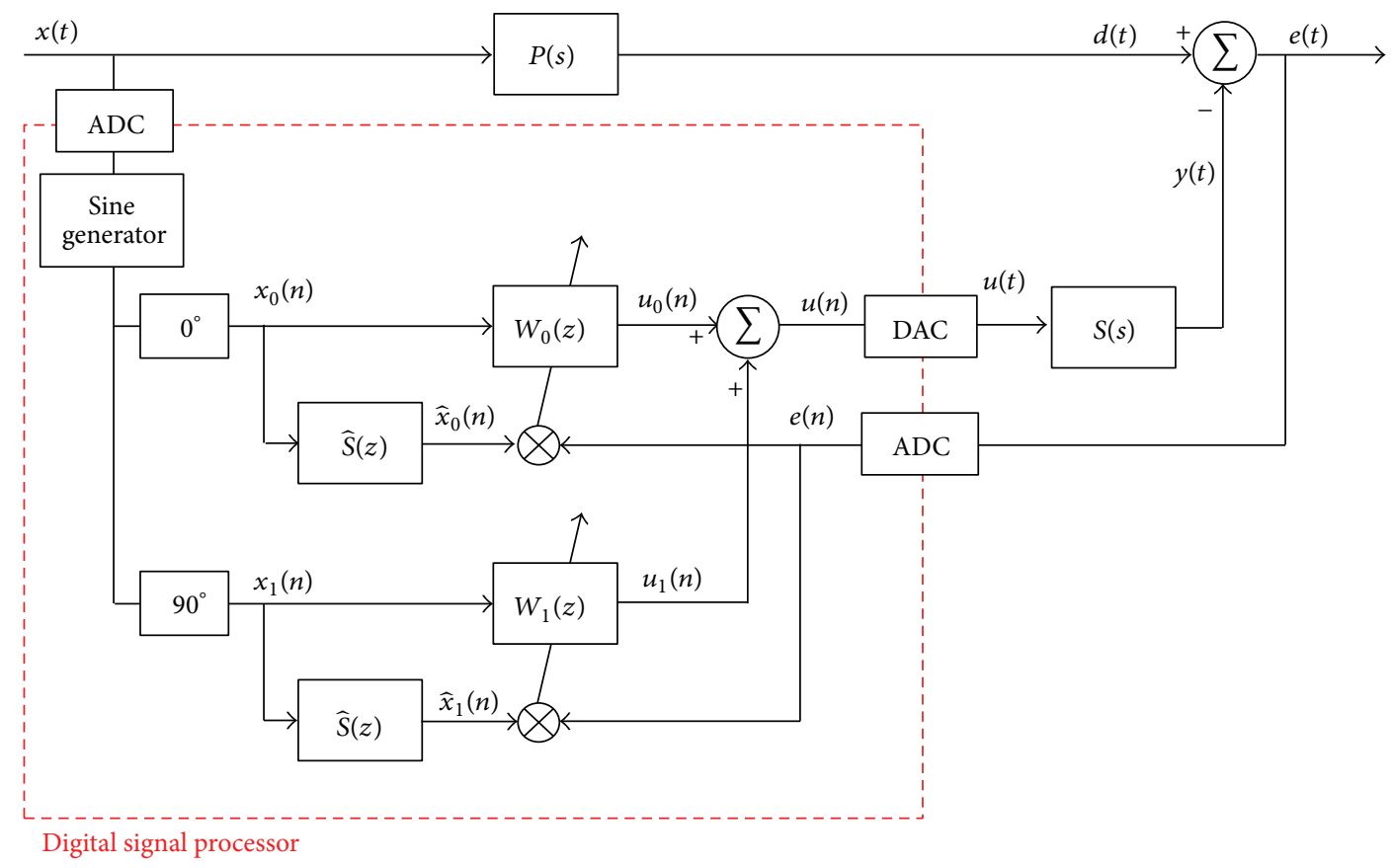

FIgURE 1: Block diagram of the narrowband FxLMS algorithm.

stability, and the computation complexity for a duct ANC system and an optimum IRF length is determined.

In Section 2, a theoretical examination is investigated for the effect of the IRF of the secondary path in the FxLMS algorithm. Section 3 shows the experimental setup for realtime ANC control of the duct system. Section 4 includes the results and analysis of the control experiment. Finally conclusions are described in Section 5.

\section{Theoretical Considerations}

Figure 1 shows a typical ANC block diagram of the SISO (single input single output) adaptive feedforward FxLMS algorithm that cancels the primary narrowband noise by the secondary source [1].

As illustrated in Figure 1, $x(t)$ is the reference signal, $d(t)$ is the disturbance signal, $u(t)$ is the control effort, $y(t)$ is the plant output and $e(t)$ is the error signal, and $P(s)$ and $S(s)$ are the primary and secondary paths, respectively. The blocks inside the rectangle with red dashed line are implemented in a DSP (digital signal processor) where $W(z)$ and $\widehat{S}(z)$ are the adaptive filter and the IRF of the secondary path model, respectively. In addition $x(n), \widehat{x}(n), u(n)$, and $e(n)$ are discrete signals to represent the reference, filtered reference, control effort, and error sequences, respectively.

Assuming 1 reference signal, 1 primary loudspeaker, 1 secondary loudspeaker, and 1 error microphone are installed in a duct system, the filtered reference sequence $\widehat{x}(n)$ can be expressed as

$$
\widehat{\mathbf{x}}_{i}(n)=\widehat{\mathbf{s}}^{T} \mathbf{x}_{i}(n-\ell), \quad(i=0,1, \ell=0 \sim L-1),
$$

where $L$ is the IRF filter length of $\widehat{S}(z)$ and $\widehat{\mathbf{s}}$ is the coefficient of the IRF filter. The update equation of an adaptive feedforward narrowband FxLMS algorithm can be written by [1-4]

$$
\mathbf{w}_{i}(n+1)=\mathbf{w}_{i}(n)+\alpha \widehat{\mathbf{x}}_{i}(n) e(n),
$$

where $\alpha$ is the convergence coefficient. The computation to obtain the control effort $u(n)$ in the DSP can be given by

$$
u(n)=w_{0}(n) x_{0}(n)+w_{1}(n) x_{1}(n) .
$$

Provided that $\widehat{S}(z)$ is approximately identical to $S(z)$, the cancellation of the primary noise can be achieved during control as the IRF of the secondary path model can compensate for the actual secondary path. However, the IRF with some error makes a certain level of residual noise during control as

$$
e(t)=d(t)-y(t) \neq 0 \text {. }
$$

Thus the accuracy of the IRF modeling of the secondary path is critically important in the implementation of an FxLMS based ANC system.

The computation complexity of the SISO FxLMS algorithm by length variation of the IRF filter can be given by Table 1, where the IRF filter length and control order number are $L$ and $C$, respectively.

\section{Experimental Setup}

The experimental setup for the narrowband SISO ANC control is displayed in Figure 2. A duct (length $=1800 \mathrm{~mm}$ ) ANC system is comprised of a primary loudspeaker at 


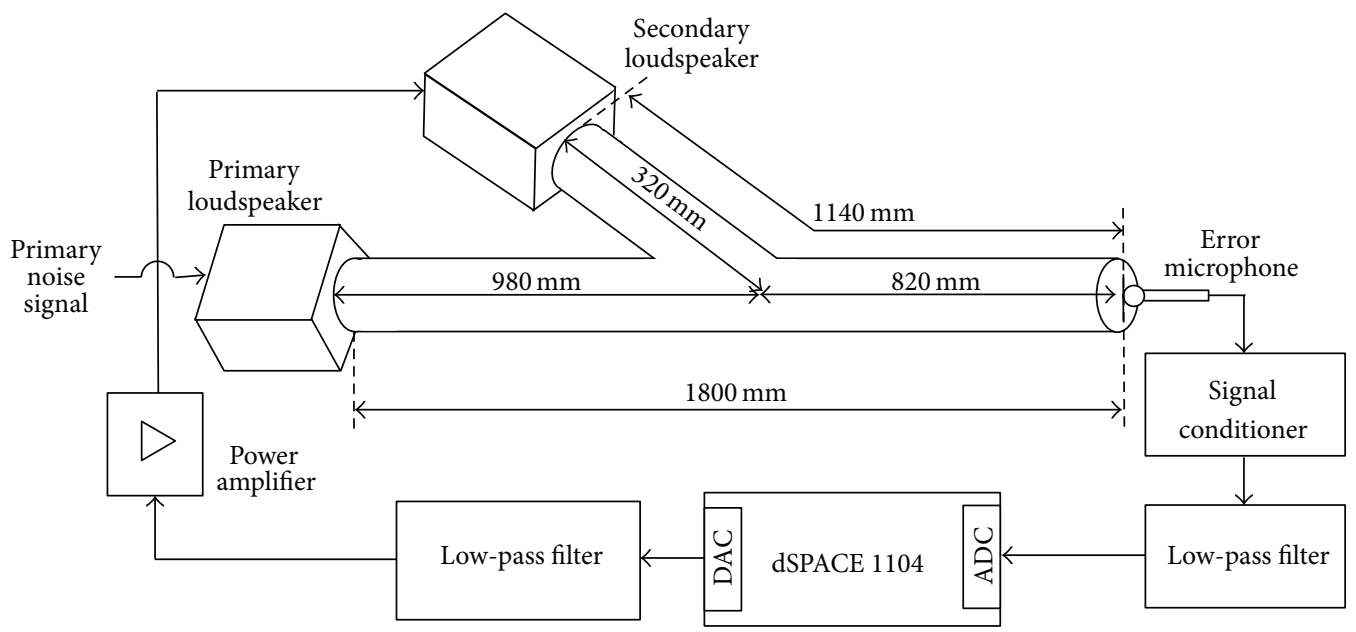

FIGURE 2: Experimental setup for the narrowband ANC for a duct.

TABLE 1: Computation complexity of the narrowband FxLMS algorithm.

\begin{tabular}{lcc}
\hline Equation & Number of additions & Number of multiplications \\
\hline$(1)$ & $2(L-1) C$ & $2 L C$ \\
$(2)$ & $2 C$ & $2 C+1$ \\
$(3)$ & $2 C-1$ & $2 C$ \\
\hline Total & $2 C(L+1)-1$ & $2 C(L+2)+1$ \\
\hline
\end{tabular}

the left end, a secondary source (control loudspeaker), an error microphone (1/2" PCB 377B11) at the right end, a power amplifier (B\&K 2716C), a PCB signal conditioner, two lowpass filters (cutoff frequency: $f_{c}=500 \mathrm{~Hz}$ ), and a dSPACE 1104 controller for the implementation of the SISO FxLMS algorithm.

The primary disturbance was a narrowband signal with some sinusoidal waves such as a powertrain interior noise of a passenger car. In this study, the largest four orders by the powertrain of the passenger car are the primary noise. The primary and secondary paths were measured at first and, after carrying out the offline modeling of the secondary path, the IRF model with various lengths was calculated and implemented in the control board. Then the real-time ANC control experiment was accomplished with each IRF model.

\section{Results and Discussions}

4.1. IRF Modeling from the Measured Secondary Path. White noise was used to measure the secondary path (secondary loudspeaker-error microphone) response when the sampling frequency was $f_{s}=6,000 \mathrm{~Hz}$. The sampling frequency was maintained the same all the time during this study. Figure 3(a) demonstrates that the IRF model from the measured secondary path response of the duct system has two peaks at 25 and 55 samples, respectively, and then decays quickly after 100 samples. The first and second peaks at the sample of 25 and 55, respectively, are the most important contributions to the IRF and indicate the time delays in the secondary path of about $4.17 \mathrm{msec}$ and $9.17 \mathrm{msec}$, respectively. As can be seen from Figure 3(a), at the sample of 400, the IRF settles down on zero and this allows assuming that the IRF length of 400 samples may represent the secondary path response accurately. Thus the digital plant model with the IRF length of $L=400, \widehat{S}_{400}(z)$, can be an approximated model of the actual plant (secondary path), $S(z)$, as follows:

$$
\widehat{S}_{400}(z) \approx S(z) \text {. }
$$

Four different IRF lengths of $L=30,63,93$, and 400 are considered and plotted in Figure 3(b) to compare their frequency response functions (FRFs). The magnitude of the FRF with $L=30$ (dashed lines) traces simply the averaged plot of the FRF with $L=400$ (thick solid lines) without resonances or antiresonances in the frequency range below $1200 \mathrm{~Hz}$ and its phase response looks almost as a straight line. For $L=63$, the magnitude response follows some main resonances and antiresonances as shown in Figure 3(b). In the case of $L=93$, the magnitude and phase responses are very similar to those of $L=400$.

The mean square error (MSE) is defined by the difference between the two plant models of $\widehat{S}_{400}(z)$ and $\widehat{S}_{L}(z)$ when the input signal $x(n)$ is a white noise and can be represented as

$$
\mathrm{MSE}=E\left[x(n)\left[\widehat{S}_{400}(z)-\widehat{S}_{L}(z)\right]\right],
$$

where $E[]$ is the expectation operator. As the MSE indicates the accuracy of the secondary path model by comparing the outputs of the actual secondary path and its model against a white noise input, the MSE is an important factor to assess the model reliability.

The MSE curve against the IRF length of $L=0-120$ is plotted in Figure 4(a) and it indicates that the MSE is dramatically reduced by the increase of the IRF length. Actually the inclusion of the peaks in the IRF of Figure 4(a) gives the most critical influences to the accuracy of modeling of the secondary path. For the best performance and robust stability, every peak is necessary in the IRF model; however 


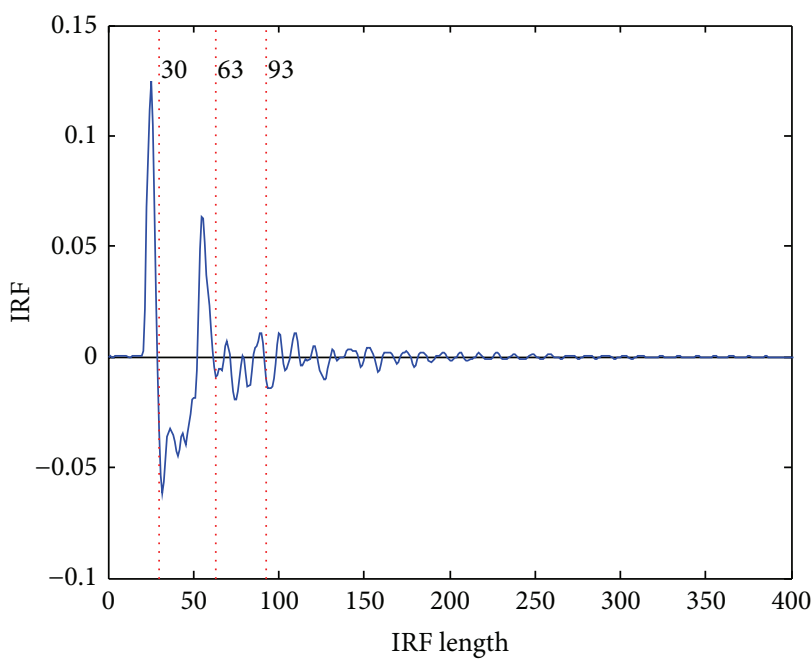

(a)
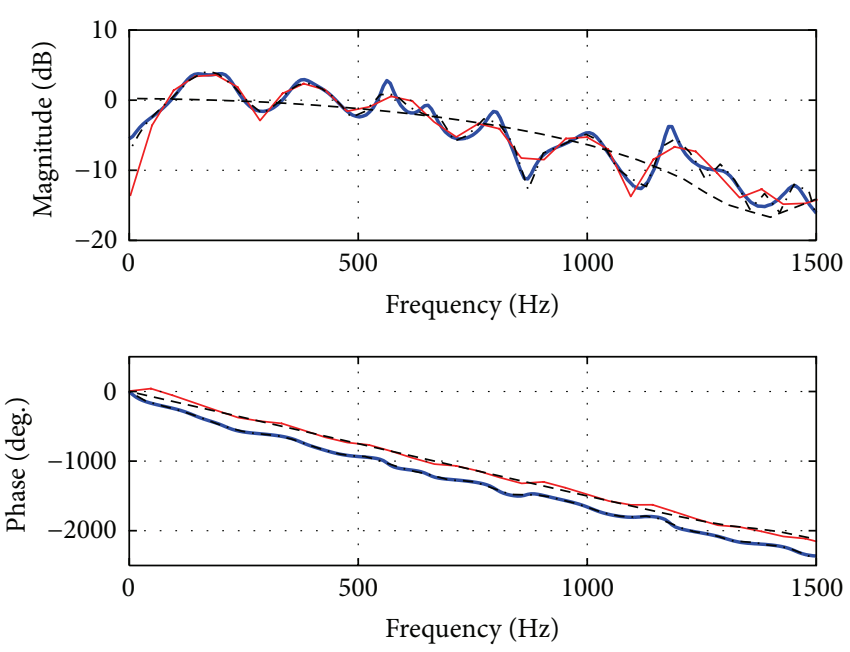

- IRF length 400

— IRF length 63

-. - IRF length 93

(b)

FIgURE 3: Plant modeling from the measured secondary path response. (a) IRF. (b) Comparison of FRFs for four different IRF lengths at $L=$ 30, 63, 93, and 400 (top: magnitude, bottom: phase).
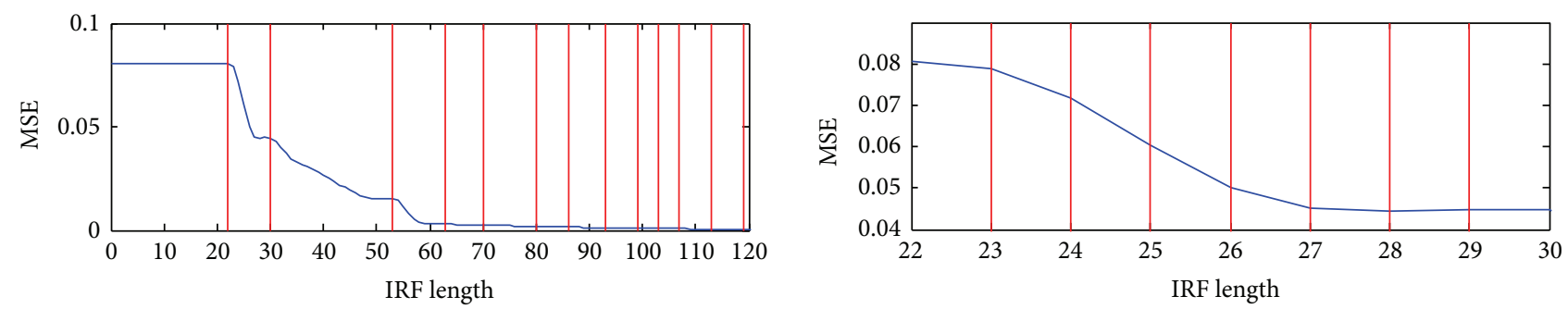

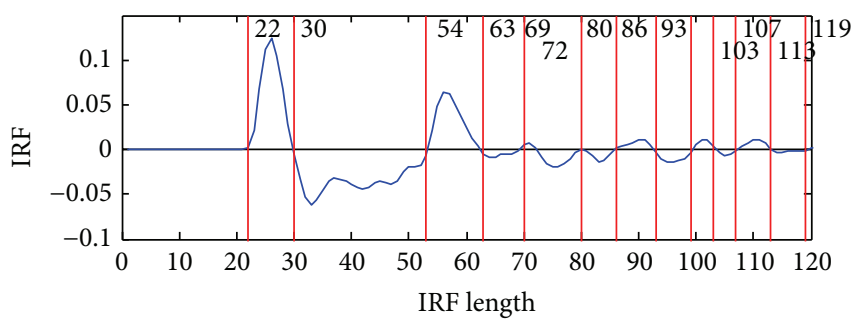

(a)

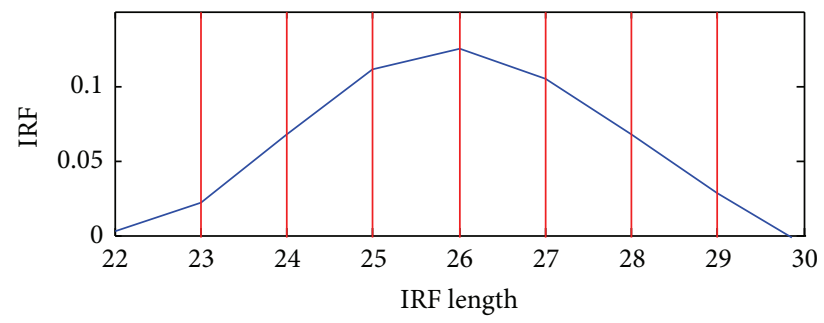

(b)

FIGURE 4: MSE against variation of the IRF length. (a) IRF length of $L=0-120$. (b) IRF length of $L=22-30$.

this can increase the computation complexity. Thus the IRF length must be chosen carefully.

There are 13 vertical lines in Figure 4(a) which implies 13 different IRF sample numbers where the IRF graph passes 0 . The MSE below $L=22$ where there is no peak in the IRF maintains its maximum level. As the IRF length increases after $L=22$, the MSE decreases accordingly as shown in Figure 4(a). Especially at $L>60$ the MSE is very low compared to that of $L=22$.

In addition, the change of the MSE against a peak in the IRF graph at $L=22-30$ is investigated in detail as illustrated in Figure 4(b) and it demonstrates that the MSE reaches the lowest level as soon as $L$ is the next sample at $L=$ 27 after the peakiest sample at $L=26$. This is important information in determining the minimized IRF length for the FxLMS algorithm particularly when the allowance of the computation complexity is limited.

4.2. Comparison of Control Performance by IRF Length Variation. Figure 5 displays the comparison of the spectrograms before and after controls with the FxLMS algorithm in the duct system of Figure 2 when the IRF length is 63 samples against a narrowband disturbance which is defined as a 3second sweep sine signal with four different orders of $\mathrm{Cl}$ 


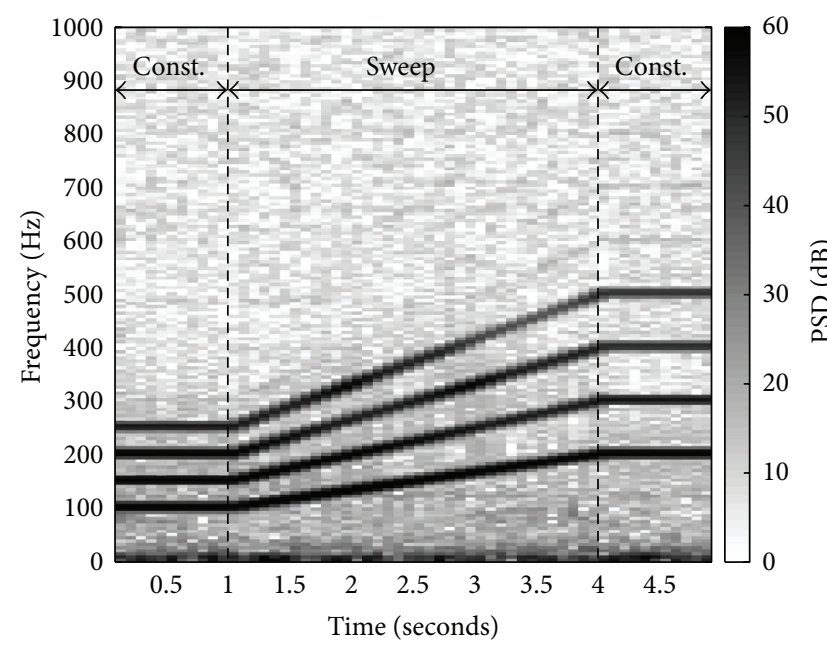

(a)

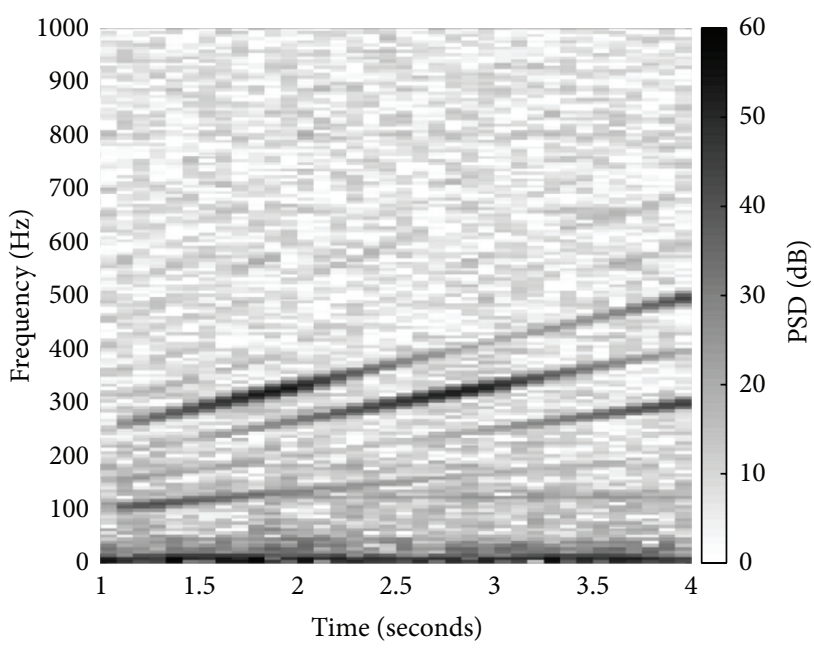

(b)

FIGURE 5: Comparison of the spectrograms before and after controls against a narrowband disturbance. (a) Spectrogram before control; (b) spectrogram after control (IRF length $L=63$ ).

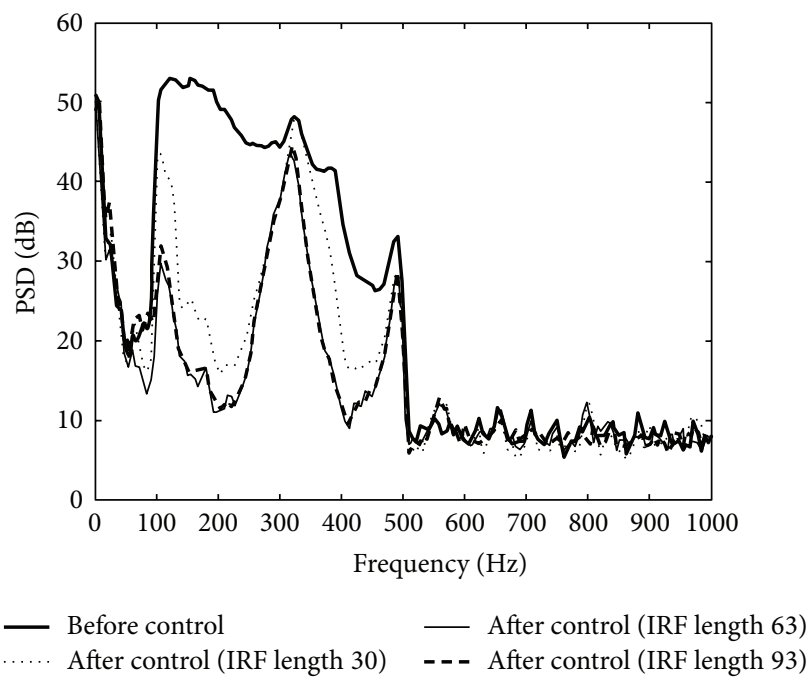

(a)

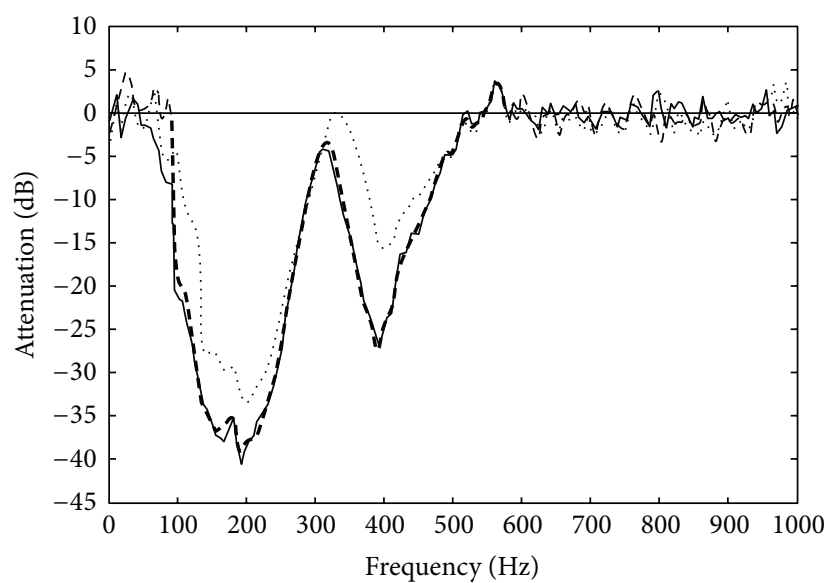

….. After control (IRF length 30) --- After control (IRF length 93) After control (IRF length 63)

(b)

FIGURE 6: Comparison of narrowband sweep real-time control performances in terms of error spectra and attenuation between before control (thick lines) and after control (IRF length $L=30$ : dotted lines, $L=63$ : thin lines, and $L=93$ : thick dashed lines). (a) Error PSD. (b) Attenuation.

$(100-200 \mathrm{~Hz}), \mathrm{C} 1.5(150-300 \mathrm{~Hz}), \mathrm{C} 2(200-400 \mathrm{~Hz})$, and C2.5 $(250-500 \mathrm{~Hz})$.

The convergence coefficient $\alpha$ defined in (2) was 0.01 for the control. The spectrogram plotted in Figure 5(a) shows that the duration of the disturbance is 5 seconds consisting of 1 second at the beginning for constant signals, 3 seconds for sweep signals, and another 1 second at the end for constant signals. As can be seen from Figure 1, the disturbance signal $d(t)$ was measured by the error microphone when the primary loudspeaker generated the narrowband signal $x(t)$ in the duct before control.

Figure 5(b) shows the spectrogram for the sweep duration (1-4 seconds) only after control with the FxLMS algorithm, as defined in Figure 1, when the IRF length of $L=63$ and indicates that an eminent reduction for every order is achieved. There are the dark parts and light parts of each line (order) after control in Figure 5(b). The dark and light parts indicate less attenuation and more attenuation, respectively. The more attenuated frequency ranges are $100-250 \mathrm{~Hz}$ and 350-450 Hz as plotted in Figures 5(b) and 6(b). Any order in the two frequency ranges was attenuated and shown lightly.

The reduction levels $(\mathrm{dB})$ in the power spectral density (PSD) after control are plotted in Figure 6 by the IRF length variation. Figure 6(a) presents the PSD comparison before control (thick solid lines) and after control for the three different IRF lengths of $L=30$ (dotted lines), 63 (thin solid lines), and 93 (thick dashed lines) samples. The PSDs after control reveal that the longer the IRF offers the further 


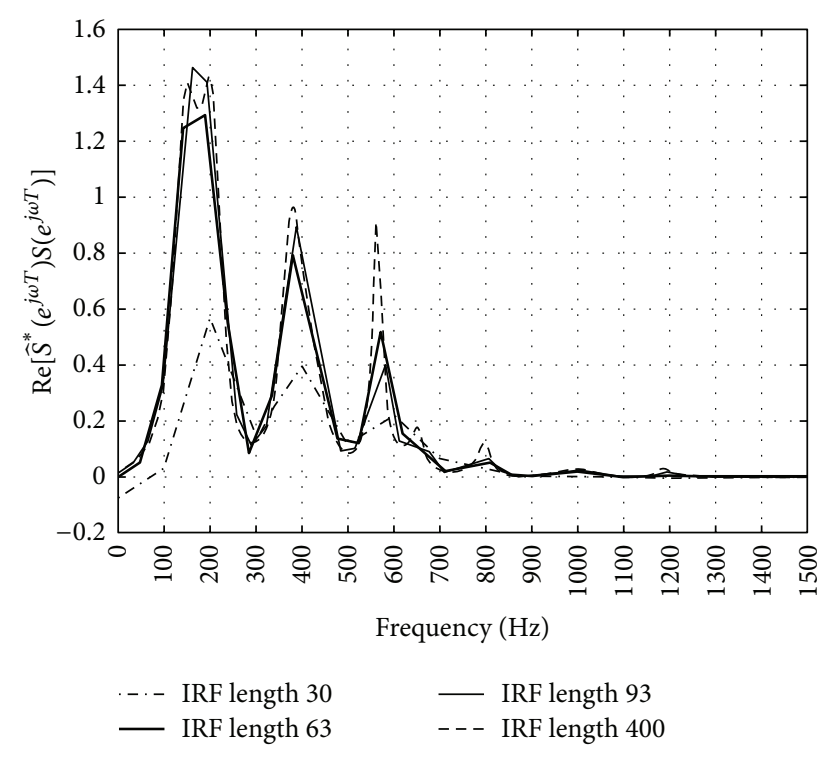

FIGURE 7: Robustness of the secondary path by the variation of the IRF length.

the reduction; however the reduction difference between the IRF lengths of $L=63$ and 93 samples is not clearly distinguished. The largest reduction achieved about 25-40 dB at $100-250 \mathrm{~Hz}$ when both $L=63$ and 93 , but the reduction at $L=30$ was $5-10 \mathrm{~dB}$ smaller than them as illustrated in Figure 6(b).

\subsection{Comparison of Robust Stability by IRF Length Variation.} It is noted from Figure 6(b) that the attenuations at some frequency ranges, such as about 100-120, 300-350, and $480-500 \mathrm{~Hz}$, are not attained properly compared to other frequencies. This suggests that, in these frequency ranges, the control stability could be easily collapsed if the convergence coefficient is increased by even a small value to achieve further attenuation. This is basically caused by the acoustical properties of the duct and can be additionally explained by the primary and secondary paths.

It is known that the stability requirement for the FxLMS algorithm can be expressed by $[1,5,6]$

$$
\operatorname{Re}\left[S\left(e^{j \omega T}\right) \widehat{S}_{L}\left(e^{j \omega T}\right)\right]>0, \quad \forall \omega T,
$$

where $\omega$ is the frequency and $T$ is the sampling interval. The stability requirement over frequency in (7) is satisfied with three different IRF lengths of $L=63$ (thick solid line), 93 (thin solid line), or 400 (dashed line) as presented in Figure 7.

But, in the case of $L=30$ (dashed and dotted line), the requirement is unsatisfied at the frequencies below $100 \mathrm{~Hz}$ and this implies the FxLMS control with $L=30$ can cause instability if the control frequency range involves below $100 \mathrm{~Hz}$. Thus the stability requirement in (7) must be considered in the determination of the IRF length in advance of control.

Figure 7 displays three outstanding peaks, assuming that $\operatorname{Re}\left[S\left(e^{j \omega T}\right) \widehat{S}_{L}\left(e^{j \omega T}\right)\right]>0.2$, at about 100-270, 320-460, and $530-600 \mathrm{~Hz}$ are the three largest resonant frequencies of the
FRF of the secondary path in Figure 3(b) and two valleys between them at about $270-320$ and $460-530 \mathrm{~Hz}$. In case of $L=30$, the frequency ranges for the peaks and valleys are partially different. The frequencies of the valleys such as around $300 \mathrm{~Hz}$ and $500 \mathrm{~Hz}$ in Figure 7 are almost identically located to the unattenuated frequencies as can be seen from Figure 6.

This coincidence discloses maintaining as higher values of $\operatorname{Re}\left[S\left(e^{j \omega T}\right) \widehat{S}_{L}\left(e^{j \omega T}\right)\right]$ as possible in the frequency range of interest and this allows higher convergence coefficients to achieve further attenuation. The valleys near zero in Figure 7 strictly restrict the increase of the convergence coefficients and this limits the control performance. This is because the increased convergence coefficient can cause more performance but less stability and if it is excessive the control system can be unstable. Thus obtaining robust stability with higher performance needs higher values of $\operatorname{Re}\left[S\left(e^{j \omega T}\right) \widehat{S}_{L}\left(e^{j \omega T}\right)\right]$ at the valleys in the frequency ranges of interest.

4.4. Comparison of Computation Complexity by IRF Length Variation. Computation complexity defined in Table 1 against various IRF lengths with the FxLMS algorithm their mean values of attenuation in the duct ANC experiment are summarized in Table 2. The IRF lengths considered in the control experiment are $L=30,54,63,70,80,86,93,99$, and 400 . Table 2 shows the attenuation is almost the same of about $-21.50 \mathrm{~dB}$ in average when $L$ is greater than or equal to 63 . Considering the computation complexity and performance by the IRF length variation, it indicates that the IRF length of $L=63$, which contains the two major peaks in the IRF graph as plotted in Figure 3(a), is the most reasonable choice in the duct system. This analysis allows relieving the computation complexity by reducing about $84 \%$ of the IRF length with the same performance as presented in Table 2.

Therefore, the IRF length variation effect of the secondary path model $\widehat{S}_{L}(z)$ is investigated in this study in terms of MSE, involvement of major peaks in the IRF graph, stability requirement, and attenuation and these terms have strong correlations with the optimum IRF length. The results in this study can be useful in various ANC applications.

In case the primary noise is broadband, it is expected that this approach can be worked well since it is based on the analysis of the secondary path.

\section{Conclusions}

This study presents the length variation effect of the secondary path IRF model in an ANC duct system. The main investigations can be described in the following.

The major peaks in the full IRF graph are needed to be involved in the IRF model for the FxLMS algorithm. Although the computation complexity is limited, the IRF model requires containing the peakiest and the next samples at least of the last major peak in the full IRF graph. The stability requirement of $\operatorname{Re}\left[S\left(e^{j \omega T}\right) \widehat{S}_{L}\left(e^{j \omega T}\right)\right]>0$ must be considered in the determination of the IRF length in 
TABLE 2: Computational complexity per sample and mean values of attenuation by the IRF length variation $(\alpha=0.01)$.

\begin{tabular}{lccc}
\hline IRF length $(L)$ & Number of additions & Number of multiplications & Mean value of attenuation $[100 \sim 500 \mathrm{~Hz}]$ \\
\hline 30 & $247(7.70 \%)$ & $257(7.99 \%)$ & $-15.01 \mathrm{~dB}$ \\
54 & $439(13.69 \%)$ & $449(13.96 \%)$ & $-20.78 \mathrm{~dB}$ \\
$\mathbf{6 3}$ & $511(\mathbf{1 5 . 9 3} \%)$ & $\mathbf{5 2 1}(\mathbf{1 6 . 2 0} \%)$ & $-\mathbf{2 1 . 4 8 ~ d B}$ \\
70 & $567(17.68 \%)$ & $577(17.94 \%)$ & $-21.57 \mathrm{~dB}$ \\
80 & $647(20.17 \%)$ & $657(20.42 \%)$ & $-21.37 \mathrm{~dB}$ \\
86 & $695(21.67 \%)$ & $705(21.91 \%)$ & $-21.63 \mathrm{~dB}$ \\
93 & $751(23.42 \%)$ & $761(23.66 \%)$ & $-21.21 \mathrm{~dB}$ \\
99 & $799(24.91 \%)$ & $809(25.15 \%)$ & $-21.37 \mathrm{~dB}$ \\
400 & $3207(100 \%)$ & $3217(100 \%)$ & - \\
\hline
\end{tabular}

advance of control. In addition, robust stability with higher performance demands higher values of $\operatorname{Re}\left[S\left(e^{j \omega T}\right) \widehat{S}_{L}\left(e^{j \omega T}\right)\right]$ at the valleys in the frequency ranges of interest.

This investigation clarifies that the most reasonable IRF length, considering the computation complexity and performance, for the implementation in an embedded control board can be determined systematically through the investigation on an ANC duct system in this study. For future study, this approach will be extended to an ANC system against a broadband primary noise.

\section{Competing Interests}

The authors declare that they have no competing interests.

\section{Acknowledgments}

This work was supported by the Incheon National University Research Grant in 2012.

\section{References}

[1] S. J. Elliott, Signal Processing for Active Control, Academic Press, New York, NY, USA, 2001.

[2] K. Lee, W. S. Gan, and S. M. Kuo, Subband Adaptive Filtering: Theory and Implementation, John Wiley \& Sons, 2009.

[3] Y. Kajikawa, W.-S. Gan, and S. M. Kuo, "Recent advances on active noise control: open issues and innovative applications," APSIPA Transactions on Signal and Information Processing, vol. 1, article e3, 2012.

[4] S. Ryu, Y. J. Park, and Y.-S. Lee, "Active suppression of narrowband noise by multiple secondary sources," Journal of Sensors, vol. 2016, Article ID 6276828, 9 pages, 2016.

[5] M. Morari and E. Zafiriou, Robust Process Control, PrenticeHall, London, UK, 1989.

[6] W. Ren and P. R. Kumar, "Adaptive active noise control: structures, algorithms and convergence analysis," in Proceedings of the Engineering for Environmental Noise Control (Inter-Noise '89), pp. 435-440, Newport Beach, Calif, USA, November 1989.

[7] P. R. Fraanje, S. J. Elliott, and M. Verhaegen, "Robustness of the filtered-X LMS algorithm - part II: robustness enhancement by minimal regularization for norm bounded uncertainty," IEEE
Transactions on Signal Processing, vol. 55, no. 8, pp. 4038-4047, 2007.

[8] A. P. Berkhoff, "A technique for improved stability of adaptive feedforward controllers without detailed uncertainty measurements," Smart Materials and Structures, vol. 21, no. 6, Article ID 064003, 2012. 


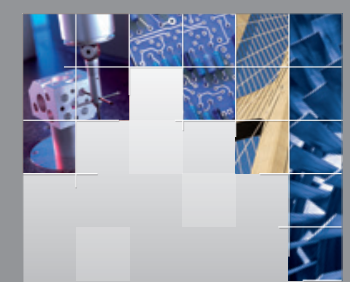

\section{Enfincering}
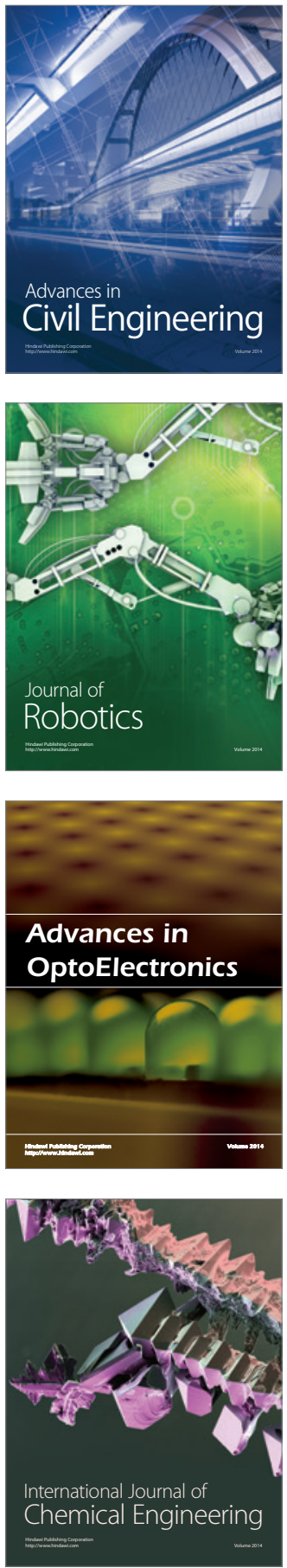

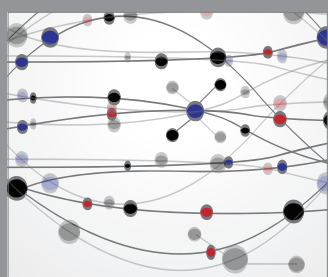

The Scientific World Journal

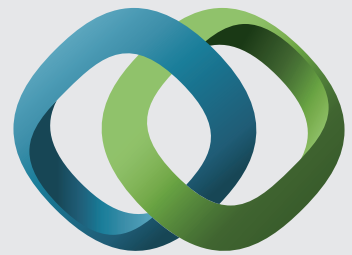

\section{Hindawi}

Submit your manuscripts at

http://www.hindawi.com
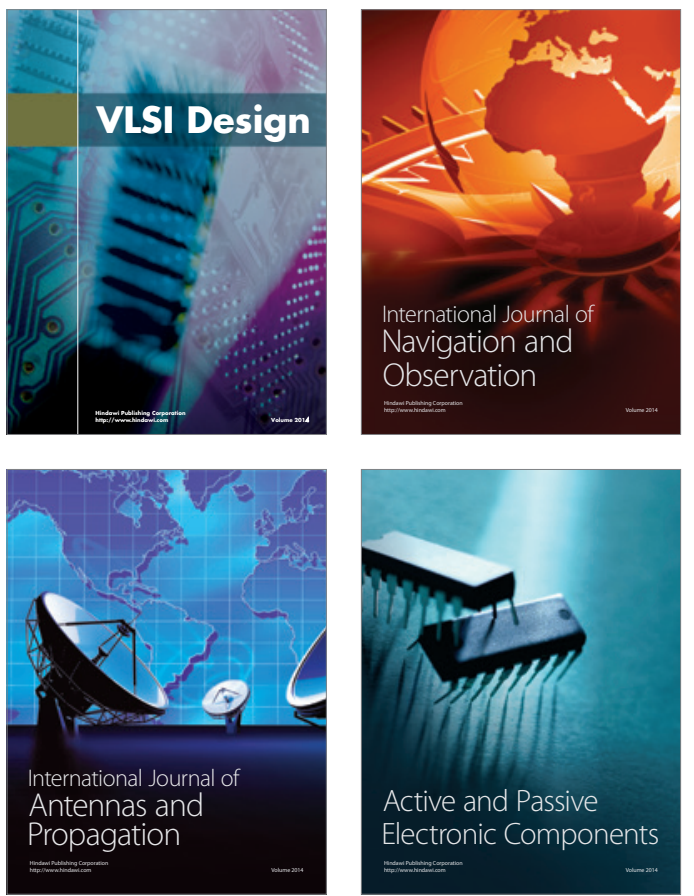
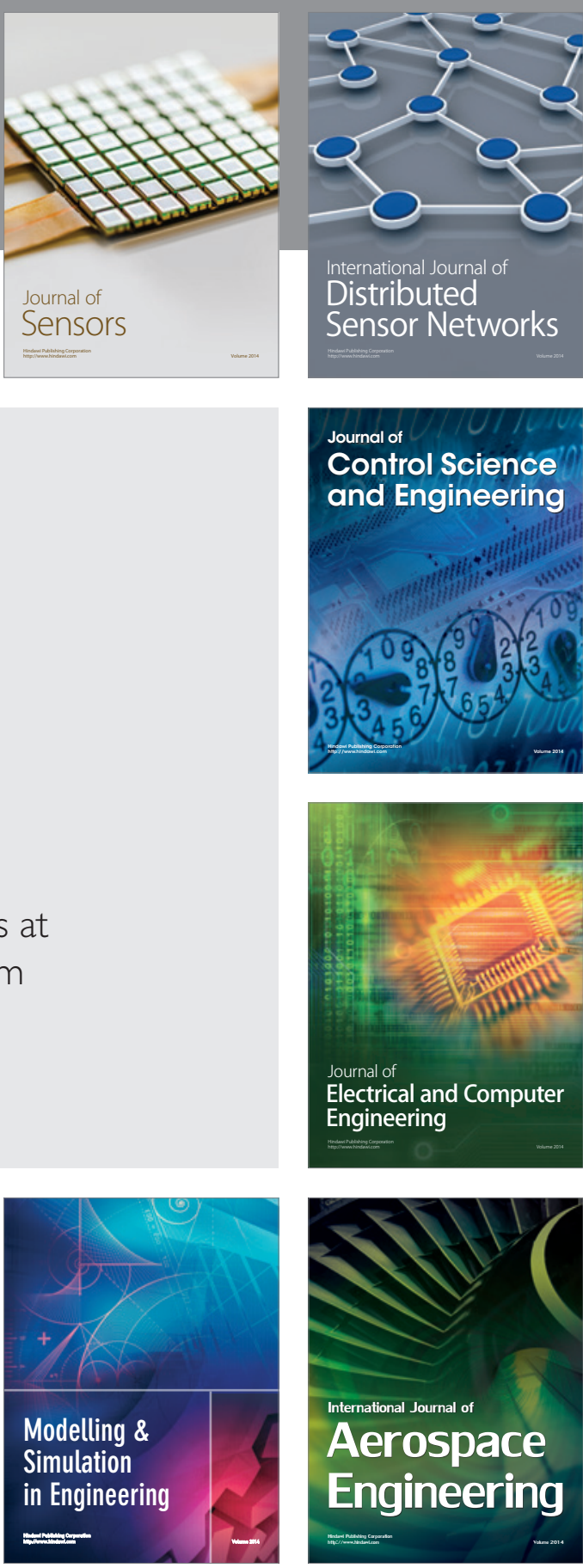

International Journal of

Distributed

Sensor Networks

Journal of

Control Science

and Engineering
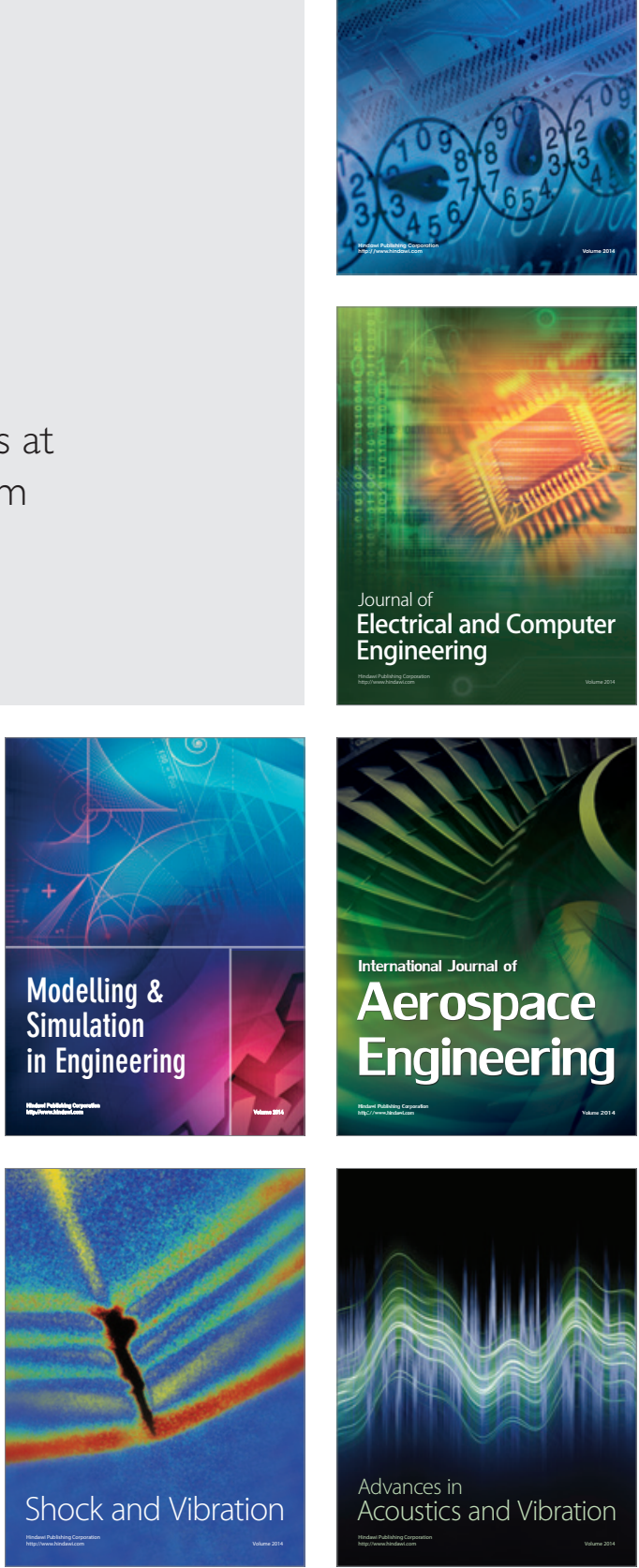